\section{Children have been left behind during the HIV 90-90-90 strategy implementation in South Africa}

To the Editor: Worldwide, children still lag in accessing HIV services, ${ }^{[1]}$ despite the Sustainable Development Goals and the UNAIDS 95-95-95 strategy highlighting the critical need for focus on children and adolescents. ${ }^{[2]}$ By 2020, only half (53\%) of HIV-infected children globally were on antiretroviral therapy (ART), compared with two-thirds $(68 \%)$ of adults. ${ }^{[1]}$ This discrepancy also exists in South Africa (SA). In 2019, among adults, 93\% had known HIV status, and $71 \%$ of those who were positive were recipients of ART, with $61 \%$ virally suppressed. Children had much lower rates: $77 \%, 63 \%$ and $63 \%$, respectively. ${ }^{[3,4]}$

Recent City of Johannesburg data indicate that children on ART had constantly lower viral load suppression rates compared with adults throughout the 90-90-90 strategy implementation period. ${ }^{[5]}$

Case-finding has received priority attention from SA health services and international funders. ${ }^{[6]}$ However, poor retention in care and inadequate viral suppression threaten programme success. Left unattended, this situation leads not only to high morbidity and mortality in children but also to the development of a cohort of young adults with high levels of drug resistance, placing control of the epidemic at risk.

Challenges to retention and adherence to ART in children include child-unfriendly ART formulations (such as unpalatable or large-volume syrups), non-availability of fixed-dose combinations, and formulations requiring refrigeration. ${ }^{[7]}$ Inadequate supervision and poor health literacy of caregivers, poor caregiver-child relationships and support systems, and non-disclosure to the child and other family members of his/ her HIV-positive status further hinder adherence. $^{[8,9]}$

We have to do more to ensure that children are initiated on ART, remain in care, and achieve virological suppression. We suggest five key interventions that can change the trajectory of HIV care for SA children (Table 1).

\section{J C Y Nyasulu}

Division of Health Systems and Public Health, Department of Global Health, Faculty of Medicine and Health Sciences, Stellenbosch University, Cape Town, South Africa; Division of Community Paediatrics, School of Clinical Medicine, Faculty of Health Sciences, University of the Witwatersrand, Johannesburg, South Africa; AFRIQUIP Health Systems, Johannesburg, South Africa julietnyasulu@gmail.com

Table 1. Recommendations to improve retention, adherence and viral suppression among children

\begin{tabular}{l} 
Intervention \\
\hline 1. Fast-tracked approval and introduction of \\
new paediatric ART formulations, ${ }^{[7]}$ including \\
simplified regimens with once-daily dosing and \\
no storage requirements. ${ }^{[10]}$
\end{tabular}

2. Community education on paediatric HIV and demand creation for services to increase acceptability of HIV testing and treatment.

3. Prioritise children for early tracing and/or linkage to care during routine ART tracing processes. $^{[7]}$

4. Investigate and validate mechanisms of lowvolume blood collection, thereby reducing the burden of paediatric phlebotomy.

5. Integrate HIV care and support interventions into community support structures, including orphan and vulnerable children programmes. ${ }^{[9}$

\section{Feasibility}

Formulations approved in other countries, and in alignment with our treatment guidelines, are available, including smaller dose $\mathrm{ABC} / 3 \mathrm{TC}$ tablet co-formulations, LPV/RTV pellets, DTG $10 \mathrm{mg}$, and others.

Mass media campaigns could change the public perception that children living with HIV do not survive and thrive.

Tracing processes include file reviews to verify missed appointments. This could identify priority patients for tracing, including children.

GeneXpert technology available for use in TB diagnostics can analyse viral load with low volumes of blood using capillary tubes.

Several community-based structures provide support services for vulnerable children.

\section{J Dunlop}

Division of Community Paediatrics, School of Clinical Medicine, Faculty of Health Sciences, University of the Witwatersrand, Johannesburg, South Africa; Anova Health Institute, Johannesburg, South Africa

\section{H Pandya, W Slemming}

Division of Community Paediatrics, School of Clinical Medicine, Faculty of Health Sciences, University of the Witwatersrand, Johannesburg, South Africa

\section{Fick}

Division of Community Paediatrics, School of Clinical Medicine, Faculty of Health Sciences, University of the Witwatersrand, Johannesburg, South Africa; Wits Reproductive Health and HIV Institute, University of the Witwatersrand, Johannesburg, South Africa

\section{H Saloojee}

Division of Community Paediatrics, School of Clinical Medicine, Faculty of Health Sciences, University of the Witwatersrand, Johannesburg, South Africa

1. Joint United Nations Programme on HIV and AIDS (UNAIDS). 2020 global AIDS update - Seizing the moment - Tackling

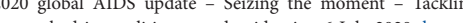
entronch whw.unaids.org/en/resources/docu

2. Joint United Nations Programme on HIV and AIDS (UNAIDS). How AIDS changed everything: MDG 6: 15 years, 15 lesson of hope from the AIDS response. 14 July 2015. https://www unaids.org/en/resources/documents/2015/MDG6_15years15lessonsfromtheAIDSresponse (accessed 13 September 2021). 3. Joint United Nations Programme on HIV and AIDS (UNAIDS). UNAIDS data 2020. 6 July 2020. https://www.unaids.org/en/ resources/documents/2020/unaids-data (accessed 13 September 2021).

4. Pillay Y. Setting the scene: Some data on the HIV epidemic. Presented at the 9th SA AIDS Conference, Durban, South Africa, 11 - 14 June 2019.

. Nyasul JCY, MePosa I Sikhathane BP, Pandya $\mathrm{H}_{\mathrm{L}}$ Access to

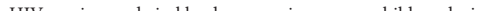
He services and viral load suppression among children during the 90-90-90 strategy implementa in Soth Afrea. A time series analysis. South Afr J HIV Med 2021 doi.org/10.4102/sajhivmed.v22i1.1187

6. Simon KR, Flick RJ, Kim MH, et al. Family testing: An index case finding strategy to close the gaps in pediatric HIV diagnosis J Acquir Immune Defic Syndr 2018;78(Suppl 2):S88-S97. https:// doi.org/10.1097/QAI.0000000000001731

. Schlatter AF, Deathe AR, Vreeman RC. The need for pediatric formulations to treat children with HIV. AIDS Res Treat 2016;2016:1654938. https://doi.org/10.1155/2016/1654938

8. Ardura-Garcia C, Feldacker C, Tweya H, et al. Early tracing of children lost to follow-up from antiretroviral treatment. I Acquir Immune Defic Syndr 2015:70(5):e160-el67. https//do Acquir Immune Defic Syndr 2015,005):

et al. The Tingathe programme: A pilot intervention using community health workers to create continuum of care in the prevention of mother to child transmission of HIV (PMTCT) cascade of services in Malawi. J Int AIDS Soc 2012;15(Suppl 2):17389. https://doi.org/10.7448 LAS.15.4.17389

10. Hien $\mathrm{H}$, Meda N, Diagbouga S, et al. 24-month adherence, tolerance and efficacy of once-a-day antiretroviral therapy with didanosine, lamivudine, and efavirenz inAfrican HIV-1 infected children: ANRS 12103/12167. Afr Health Sci 2013;13(2):287294. https://doi.org/10.4314/ahs.v13i2.13

S Afr Med J 2021;111(10):921. https://doi.org/10.7196/ SAMJ.2021.v111i10.15915 